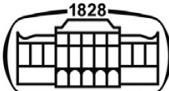

AKADÉMIAI KIADÓ

\title{
Myocardial perfusion imaging using computed tomography: Current status, clinical value and prognostic implications
}

\section{IMAGING}

\section{REVIEW ARTICLE}

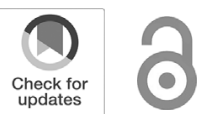

IMAGING 13 (2021) 1, 49-60

DOI: $10.1556 / 1647.2020 .00009$

(c) 2020 The Author(s)

${ }^{*}$ Corresponding author. Tel.: +3620 6663857.

E-mail: bori.vattay@gmail.com

\author{
BORBÁLA VATTAY* ๑, MELINDA BOUSSOUSSOU, \\ SAROLTA BORZSÁK, MILÁN VECSEY-NAGY, JUDIT SIMON, \\ MÁRTON KOLOSSVÁRY, BÉLA MERKELY and \\ BÁLINT SZILVESZTER
}

MTA-SE Cardiovascular Imaging Research Group, Heart and Vascular Center, Semmelweis University, Budapest, Hungary

Received: August 27, 2020 • Accepted: November 18, 2020

\begin{abstract}
Combined anatomical and functional evaluation of coronary artery disease (CAD) using computed tomography (CT) has recently emerged as an accurate, robust, and non-invasive tool for the evaluation of ischemic heart disease. Cardiac CT has become a one-stop-shop imaging modality that allows the simultaneous depiction, characterization, and quantification of coronary atherosclerosis and the assessment of myocardial ischemia. Advancements in scanner technology (improvements in spatial and temporal resolution, dual-energy imaging, wide detector panels) and the implementation of iterative reconstruction algorithms enables the detection of myocardial ischemia in both qualitative and quantitative fashion using low-dose scanning protocols. The addition of CT perfusion (CTP) to standard coronary CT angiography is a reliable tool to improve diagnostic accuracy. CTP using static first-pass imaging enables qualitative assessment of the myocardial tissue, whereas dynamic perfusion imaging can also provide quantitative information on myocardial blood flow. Myocardial tissue assessment by CTP holds the potential to refine risk in stable chest pain or microvascular dysfunction. CTP can aid the detection of residual ischemia after coronary intervention. Comprehensive evaluation of CAD using CTP might therefore improve the selection of patients for aggressive secondary prevention therapy or coronary revascularization with high diagnostic certainty. In addition, prognostic information provided by perfusion CT imaging could improve patient outcomes by quantifying the ischemic burden of the left ventricle. The current review focuses on the clinical value of myocardial perfusion imaging by CT, current status of CTP imaging and the use of myocardial CTP in various patient populations for the diagnosis of ischemic heart disease.
\end{abstract}

\section{KEYWORDS}

myocardial CT perfusion, cardiac CT, ischemic heart disease

\section{Introduction}

The evaluation of symptomatic patients suggestive of myocardial ischemia is fundamental challenge in clinical care. The use of functional imaging in cardiology has led to a high number of unnecessary catheterizations with no obstructive disease [1]. On the other hand, anatomical evaluation by using coronary computed tomography angiography (CTA) has emerged as a reliable and accurate diagnostic tool for the evaluation of coronary artery disease $(\mathrm{CAD})[2,3]$. Due to its high sensitivity and negative predictive value it is an accurate tool to rule out CAD in patients with low-to intermediate risk of obstructive disease $[4,5]$, however finding the proper test for the assessment of patients with suspected CAD without generating unnecessary downstream testing and to guide patient management is challenging. 
Limitations of coronary CTA include that it tends to overestimate the severity of stenosis especially in the case of intermediate to severe lumen stenosis, multivessel disease, and extensive calcification, consequently lowering its specificity and positive predictive value. CT perfusion (CTP) imaging has emerged as a robust tool to complement traditional anatomical assessment of CAD by CTA. CTP imaging has the potential to also improve diagnosis in patients with higher likelihood of CAD or with microvascular disease and to detect residual ischemia after percutaneous coronary intervention. Current review focuses on the clinical value of myocardial perfusion imaging by CT, current status of CTP imaging and the use of myocardial CTP in various patient populations for the diagnosis of ischemic heart disease.

\section{Coronary CTA for the evaluation of stable chest pain: current status}

In the 2019 European Society of Cardiology guidelines CTA received class I recommendation for the evaluation of chronic coronary syndrome in patients with stable symptoms [6]. Two main studies were performed to underline the pivotal role of anatomical testing using CTA for stable angina. The results of the randomized SCOT-HEART (Scottish Computed Tomography of the Heart, NCT01149590) trial have shown that CTA based patient management could substantially improve long term outcomes as compared to standard care alone [7]. The PROMISE (Prospective Multicenter Imaging Study for Evaluation of Chest Pain, NCT01174550) trial has evaluated 10,003 symptomatic patients who were randomized either to functional or anatomical strategy to prove that CTA is noninferior to functional testing [8]. Although the trial found no difference in the primary outcome, it did emphasize the prognostic value of CTA findings. Importantly, CTA also permits the detection of prognostically relevant nonobstructive CAD (between 1 and 69\% luminal narrowing) that has been identified as an important predictor of adverse cardiac events [9]. Anatomical assessment using CTA provided better prognostic information as compared with functional testing that resulted also from the indiscriminatory nature of ischemia testing.

Despite its excellent capability to detect atherosclerotic plaque burden, the physiologic significance of the identified lesions remains unknown. In the FAME (Fractional Flow Reserve versus Angiography for Multivessel Evaluation, NCT00267774) study combining anatomical and functional evaluation (invasive measurement of lesion specific ischemia) of patients with suspected CAD improved clinical outcome [10]. Similarly, the combination of coronary CTA and CT perfusion imaging can improve diagnostic accuracy and proper identification of patients who require revascularization. The ISCHEMIA (International Study of Comparative Health Effectiveness with Medical and Invasive Approaches, NCT01471522) trial further improved our understanding on the management of patients with stable angina and proven ischemia [11]. Optimal medical therapy proved to be non-inferior to percutaneous intervention for adverse events, although latter could improve symptoms of angina. Notably, in the ISCHEMIA trial anatomical severity of CAD increased risk for adverse events, whereas proven ischemia did not.

\section{CT perfusion imaging for the detection of myocardial ischemia}

As an anatomical imaging test, traditional coronary CTA does not allow the detection of myocardial ischemia. Physiological evaluation of CAD is of utmost importance for patient management since ischemia driven revascularization could provide better outcomes [12-15]. Also, there is a remarkable discrepancy between luminal stenosis and detectable ischemia by either single-photon emission computed tomography (SPECT) or invasive fractional flow reserve (FFR). In the RICPORD (Does Routine Pressure Wire Assessment Influence Management Strategy at Coronary Angiography for Diagnosis of Chest Pain? NCT01070771) study after visual assessment of coronary stenosis by invasive coronary angiography (ICA), additional FFR measurement changed patient management in onequarter of patients with stable angina [16]. Similarly, only $50 \%$ of patients with obstructive CAD on CTA had abnormal perfusion parameters assessed with SPECT [17].

Patients with less than $30 \%$ stenosis would possibly not benefit from ischemia testing, whereas lesions above $80 \%$ stenosis proved to be predominantly hemodynamically relevant [18]. Notably, revascularization does not improve symptoms or outcome in patients with no detectable ischemia, whereas some evidence suggests that more than $10 \%$ ischemia is associated with better prognosis after revascularization [19].

Functional testing includes stress echocardiography, stress magnetic resonance imaging (MRI), SPECT or positron-emission tomography (PET) imaging based on local expertise and accessibility. CTP imaging was introduced as a new-comer modality for ischemia detection. CTP is able to detect hypo-attenuated areas corresponding to myocardial injury in the left ventricle (LV) during first-pass of the contrast agent (see Central illustration). Advancements in CT scanner technology led to an improved spatial and temporal resolution with lower radiation exposure and contrast agent use by implementing iterative reconstruction algorithms. Also, wide detector CT scanners provide complete coverage (up to $16 \mathrm{~cm} z$-axis coverage) of the heart volume. Rest CTA images are performed either as first to rule out $\mathrm{CAD}$ with subsequent ischemia provocation using vasodilator stressor agents (predominantly adenosine and regadenoson) or after stress imaging to avoid pre-enhancement of the myocardial tissue [20]. There are no guidelines on the exact order of stress and rest imaging, however in general it is recommended to start with a rest scan for lower 


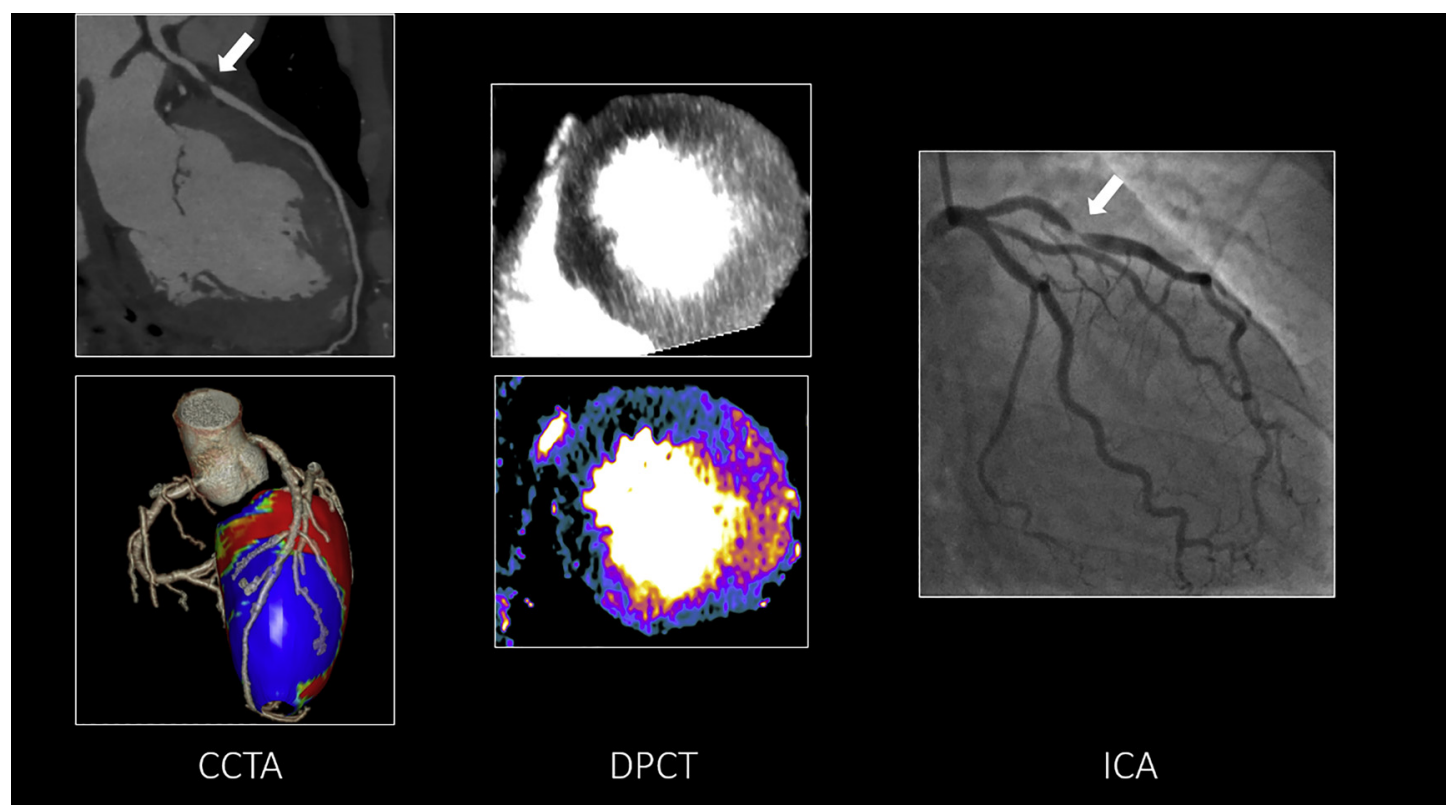

Central illustration. The addition of CTP to CCTA for the detection of hemodynamically relevant coronary lesions. Representative case of a patient presenting with stable chest pain. Panel left: CTA image of the left anterior descending (LAD) with high-grade stenosis in the proximal segment (white arrow). Panel middle: Dynamic CTP image showing hypoattenuated regions in the mid-anterior and midanteroseptal segments of the left ventricle. Panel right: ICA shows significant stenosis in LAD (white arrow). CCTA: coronary CT angiography; CTA: CT angiography; CTP: CT perfusion; DPCT: dynamic perfusion CT; ICA: invasive coronary angiography; LAD: left anterior descending

risk patients, and conversely, an initial stress scan should be performed for higher risk patients based on traditional risk scores and/or coronary calcium scoring. Notably, different stressor agents are in use to provoke ischemia during the CT scan. Recent investigations used regadenoson - a selective A2A agonist - in a single 10 second bolus that can be used in the same intravenous line as for the contrast injection [21]. Regadenoson provides adequate long and effective vasodilator stress for CTP and can be safely used without dose modulations for patients' size or co-morbidities [22].

Currently two different acquisitions are available to perform CTP with the aim to diagnose perfusion abnormalities through the myocardial tissue: static and dynamic myocardial perfusion CT. Furthermore, static stress CTP imaging can be performed using monoenergetic or dualenergy CT acquisition, where rest images are derived from the coronary CTA. Static CTP shows the peak blood flow of the myocardium (myocardial blood flow in one specific timepoint) allowing qualitative and semi-quantitative analysis of myocardial perfusion (Fig. 1). Therefore, the optimal timing for static CTP is crucial [20, 23]. Investigations demonstrated that dual-energy perfusion imaging using iodine mapping improves the detection of ischemia as compared to monoenergetic static CTP $[24,25]$. On the other hand, dynamic CTP (usually stress imaging only) depicts contrast distribution in the LV wall during several cardiac cycles in order to obtain time attenuation curves (TACs) and the arterial input function curve (upslope method) to calculate myocardial blood flow. TAC curves for normal and ischemic myocardial segments differ markedly
[26] (Fig. 2). It therefore also enables assessment of semiquantitative and quantitative parameters of myocardial perfusion such as the upslope, peak enhancement, time to peak (TTP), area under the curve, absolute, and relative myocardial blood flow (MBF) values, and myocardial blood volume (MBV). Dynamic stress CTP also allows for more precise and reproducible detection of balanced ischemia in multivessel disease as compared with static CTP. Although growing body of evidence suggest the improved diagnostic accuracy of CTP using dynamic stress perfusion protocols with quantitative assessment, this method is associated with increased radiation exposure (approx. 4-15 mSv) [20, 27]. Importantly, wide variability exists in both the imaging protocols and the cut-off values of perfusion parameters for discriminating ischemic myocardial segments. Previous studies reported a wide range of $\mathrm{MBF}$ thresholds for the detection of myocardial ischemia from 75 to even $103 \mathrm{~mL} /$ $100 \mathrm{~mL} / \mathrm{min}$ measured using a region of interest in a given segment [27-35]. Also, data are limited on the prognostic value of quantitative CTP parameters. Vendor specific technical aspects of CTP protocols are summarized in prior publications [18, 20, 24, 36]. Detailed description of technical aspects and outcomes are summarized in Table 1.

Earlier this year, the Society of Cardiovascular Computed Tomography (SCCT) released a consensus document on myocardial perfusion imaging [37]. This long-anticipated document summarizes the technical principles, diagnostic value, patient selection, image acquisition and interpretation of CTP imaging and also defines the key elements of reporting the results for the referring physician. Notably, the 


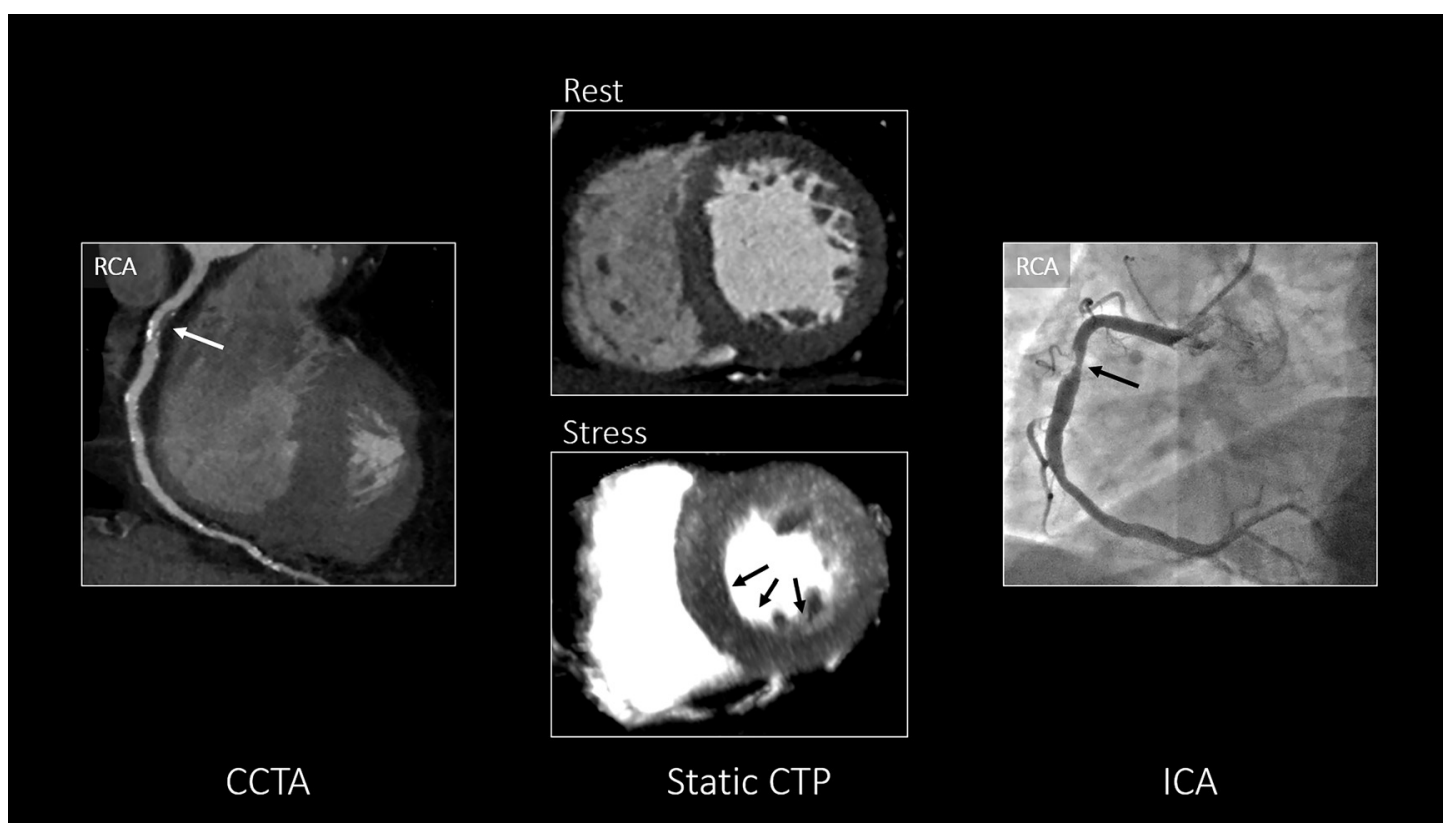

Fig. 1. Static CT perfusion imaging for detecting hemodynamically significant CAD in patients with stable chest pain and intermediate lesion. Representative images of a patient presenting with stable chest pain. Static CT perfusion allows for the visual assessment (qualitative or semi-quantitative) of perfusion defects. Panel left: CTA image of the right coronary artery (RCA) with moderate stenosis in the proximal segment (white arrow). Panel middle: Rest and stress static CTP images. No perfusion defect was observed on the rest CT scan, while hypoattenuated regions in the mid-inferior and mid-inferoseptal segments of the left ventricular wall was present under stress (black arrows). Panel right: ICA shows significant stenosis in the proximal RCA with an invasive FFR value of 0.72 (black arrow). CAD: coronary artery disease; CCTA: coronary CT angiography; CTA: CT angiography; CTP: CT perfusion; FFR: fractional flow reserve; ICA: invasive coronary angiography; RCA: right coronary artery

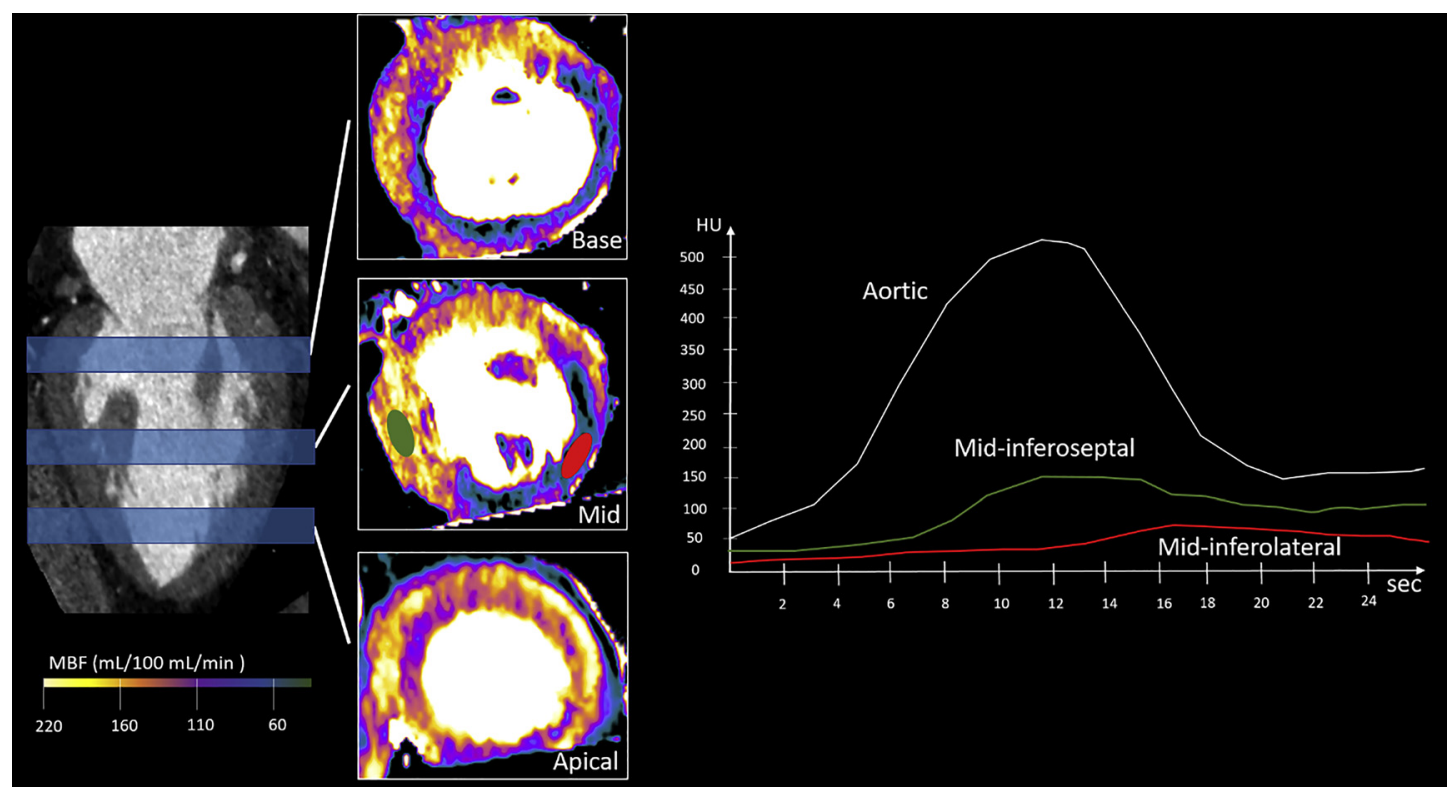

Fig. 2. Dynamic CT perfusion for the detection of myocardial ischemia. Dynamic CT perfusion enables the quantification of myocardial hemodynamics during stress. TAC curves are generated for the aorta and the selected myocardial segments. The red region of interest (ROI) represents the mid-inferolateral segment, whereas the green ROI stands for the mid-inferoseptal segment. Substantial differences are observed in the TAC curves and CTP revealed perfusion deficit of the inferolateral wall (reduced MBF values of $61 \mathrm{~mL} / 100 \mathrm{~mL} / \mathrm{min}$ in the inferolateral and normal perfusion of $143 \mathrm{~mL} / 100 \mathrm{~mL} / \mathrm{min}$ in the inferoseptal segment). CTP: CT perfusion; HU: Hounsfield unit; MBF: myocardial blood flow; TAC: time attenuation curve; ROI: region of interest 
Table 1. Technical aspects and main outcomes of static and dynamic CTP studies

\begin{tabular}{|c|c|c|c|c|c|c|c|}
\hline Author, year & Patient number & CT scanner & Stress agent & Analysis & Reference standard & Radiation dose of CTP (mSv) & Outcome \\
\hline \multicolumn{8}{|l|}{ Static CTP } \\
\hline Bettencourt et al. [70] & 101 & 64-slice & Adenosine & Visual & FFR, MRI & $5.0^{*}$ & $\begin{array}{l}\text { CTA + CTP for detecting significant CAD: SE: } \\
\text { 90\%; SP: } 81 \% \text {; PPV: } 80 \% \text {; NPV: } 90 \%\end{array}$ \\
\hline Rochitte et al. [71] & 381 & 320-slice & Adenosine & Semi-quantitative & SPECT & 5.31 & $\begin{array}{c}\text { CTA + CTP: SE: 80\%; SP: 74\%; PPV: 65\%; NPV: } \\
86 \%\end{array}$ \\
\hline Cury et al. [43] & 110 & Multivendor & Regadenoson & Semi-quantitative & SPECT & $17.70^{*}$ & $\begin{array}{c}\text { CTP was non-inferior; agreement rate: 0.87; SE: } \\
\text { 90\%; SP: } 84 \%\end{array}$ \\
\hline Pontone et al. [46] & 147 & 256-slice & Adenosine & Visual & FFR & 2.5 & $\begin{array}{l}\text { CCTA + CTP patient based SE: } 98 \% \text {; SP: } 87 \% \text {; } \\
\text { NPV: } 99 \% \text {; PPV: } 86 \%\end{array}$ \\
\hline Andreini et al. [72] & 150 & 256-slice & Adenosine & Visual & QCA & 2.26 & $\begin{array}{l}\text { Highest diagnostic accuracy using CTP + CTA } \\
95.8 \% \text { in the territory-based analysis }\end{array}$ \\
\hline \multicolumn{8}{|l|}{ Static dual-energy CTP } \\
\hline Delgado et al. [73] & 56 & DS $2 \mathrm{G}$ & Adenosine & Iodine map & MRI & 5.2 & $\begin{array}{c}\text { SE: 76\%; SP: 99\%; PPV: 89\%; NPV: 98\% per } \\
\text { segment analysis }\end{array}$ \\
\hline Meinel et al. [74] & 55 & DS $2 \mathrm{G}$ & Adenosine & Iodine map & SPECT & 7.1 & $\begin{array}{l}\text { SE: 99\%; SP: 97\%; PPV: 92\%; NPV: 100\% per } \\
\text { segment analysis }\end{array}$ \\
\hline Kim et al. [75] & 50 & DS $2 \mathrm{G}$ & Adenosine & Iodine map & MRI & 6.5 & $\begin{array}{l}\text { SE: 77\%; SP: 94\%; PPV: 53\%; NPV: 98\% per } \\
\text { segment analysis }\end{array}$ \\
\hline Ko et al. [76] & 100 & DS $1 \mathrm{G}$ & Adenosine & Iodine map & $\mathrm{QCA}+\mathrm{MRI}$ & 4.2 & $\begin{array}{c}\text { SE: } 87 \% \text {; SP: 79\%; PPV: 71\%; NPV: } 91 \% \text { vessel } \\
\text { based analysis }\end{array}$ \\
\hline \multicolumn{8}{|l|}{ Dynamic CTP } \\
\hline Bamberg et al. [30] & 31 & DS $2 \mathrm{G}$ & Adenosine & Quantitative & MRI & 11.08 & $\begin{array}{c}\text { Estimated MBF threshold for perfusion defect } 88 \\
\mathrm{~mL} / \mathrm{mg} / \mathrm{min} \text {; SE: } 77.8 \% \text {; SP: } 75.4 \% \text {; PPV: } 50.6 \% \text {; } \\
\text { NPV: } 91.3 \%\end{array}$ \\
\hline Rossi et al. [29] & 80 & DS $2 \mathrm{G}$ & Adenosine & Quantitative & FFR & 9.4 & $\begin{array}{l}78 \mathrm{~mL} / 100 \mathrm{~mL} / \mathrm{min} \text { cut-off value for MBF index; } \\
\text { SP was } 89 \% \text { for intermediate lesions }\end{array}$ \\
\hline Coenen et al. [32] & 74 & DS 3G & Adenosine & Quantitative & ICA/FFR & 9.3 & $\begin{array}{l}\text { CT MPI (indexed MBF) and CT-FFR had } \\
\text { comparable accuracy }\end{array}$ \\
\hline Nishiyama et al. [78] & 38 & 256-slice & Adenosine & Quantitative & ICA/FFR & 10.2 & $\begin{array}{l}\text { MBF cutoff } 1.26 \mathrm{~mL} / \mathrm{g} / \mathrm{min} \text { for detecting } \\
\text { obstructive CAD }\end{array}$ \\
\hline Yi et al. [79] & 60 & DS 3G & Adenosine & Quantitative & ICA/FFR & $\mathrm{NA}$ & $\begin{array}{l}\text { Relative MBF ratio with highest segmental MBF } \\
\text { provided optimal diagnostic accuracy (versus } \\
\text { average and 3rd quartile segmental MBF) }\end{array}$ \\
\hline Yang et al. [80] & 82 & DS $2 \mathrm{G}$ & Adenosine & Quantitative & ICA/FFR & 3.5 & $\begin{array}{c}\text { AUC was } 0.91 \text { for the combination of stenosis } \\
\geq 50 \% \text { by CTA and SFR (ratio of hyperemic MBF } \\
\text { in a stenosed artery versus in a non-diseased } \\
\text { artery) }\end{array}$ \\
\hline Pontone et al. [28] & 85 & 256-slice & Adenosine & Quantitative & ICA/FFR & 5.3 & $\begin{array}{c}\text { The sequential strategy of CCTA }+ \text { FFRCT }+ \\
\text { CTP showed the highest AUC }(0.919 ; P<0.05) \\
\text { as compared with all other strategies. Threshold } \\
\text { for absolute } \mathrm{MBF} 101 \mathrm{~mL} / 100 \mathrm{~g} / \mathrm{min}\end{array}$ \\
\hline Alessio et al. [81] & 34 & 256-slice & Regadenoson & Quantitative & PET & 8.4 & $\begin{array}{l}\text { Global MBF highly correlated with PET ( } r= \\
0.92 \text {; } P<0.001 \text { ); mean difference: } 0.7 \pm 26.4 \%\end{array}$ \\
\hline Yi et al. [82] & 60 & DS 3G & Adenosine & Quantitative & ICA/FFR & $\mathrm{NA}$ & $\begin{array}{l}\text { Absolute MBF value was superior than relative } \\
\text { MBF ratio (AUC:0.955 versus } 0.906 P=0.02 \text { ); } \\
\text { cut-off for absolute MBF: } 115.7 \mathrm{~mL} / 100 \mathrm{~mL} / \mathrm{min}\end{array}$ \\
\hline Li et al. [83] & 62 & DS 3G & Adenosine & Quantitative & FFR & 3.0 & $\begin{array}{l}\text { AUC: } 0.942 \text { for absolute MBF and AUC: } 0.956 \\
\text { for relative MBF lesion based analysis }\end{array}$ \\
\hline
\end{tabular}

Radiation dose for both rest and stress CTA.

1G: first-generation scanner; 2G: second-generation scanner; 3G: third-generation scanner; AUC: area under the curve; CAD: coronary artery disease; CTA: CT angiography; CCTA: coronary CT angiography; CTP: CT perfusion; DS: dual-source scanner; FFR: fractional flow reserve; MBF: myocardial blood flow; MPI: myocardial perfusion imaging; MRI: magnetic resonance imaging; mSv: millisievert; NA: non-assessable; NPV: negative predictive value; PET: positron emission tomography; PPV: positive predictive value; QCA: quantitative coronary angiography; SE: sensitivity; SFR: stress myocardial blood flow ratio; SP: specificity; SPECT: single-photon emission computed tomography. 
consensus document encourages the use of CTP in patients with high likelihood of ischemic heart disease, known CAD, prior coronary intervention or extensive calcification.

\section{Diagnostic performance of different CTP imaging protocols}

CTP is an emerging technology that was developed to improve the diagnostic performance of coronary CTA. In single center studies CTP had excellent diagnostic performance compared to SPECT, CMR, PET, ICA, and invasive FFR [38-41]. In the CORE320 (Combined Non-invasive Coronary Angiography and Myocardial Perfusion Imaging Using 320 Detector Computed Tomography, NCT00934037) multicenter study static stress myocardial CTP and SPECT perfusion were compared in patients with known significant CAD detected by ICA and found higher overall diagnostic performance for static CTP [42]. In another multicenter study Cury et al. found that CTP was non-inferior to SPECT in the detection of reverse myocardial ischemia [43]. According to the meta-analysis of Takx et al., CTP had a pooled sensitivity and specificity of 88 and $80 \%$ using ICA with FFR as reference standard [44]. Furthermore, the diagnostic performance of CTP was similar to PET and stress MRI and higher than SPECT and echocardiography. A meta-analysis of CTP studies found similar diagnostic accuracy for static and dynamic techniques with a sensitivity and specificity of 82 and $78 \%$ for static and 77 and $89 \%$ for dynamic CTP. Sørgaard et al. in their meta-analysis found that compared to coronary CTA alone, combining CTP, and coronary CTA improved specificity from 62 to $84 \%$ when using ICA as reference standard [45]. CTP is therefore a promising and accessible tool that has superior spatial resolution as compared with SPECT to detect even smaller territories of myocardial ischemia. While there are several alternative, extensively validated modalities to provide functional information on CAD, CT is the only non-invasive modality for the combined assessments of morphology and function to guide patient management.

The PERFECTION (Comparison Between Stress Cardiac Computed Tomography Perfusion Versus Fractional Flow Reserve Measured by Computed Tomography Angiography In the Evaluation of Suspected Coronary Artery Disease) prospective study aimed to compare the diagnostic accuracy of CTA with combined CTA + CT-FFR and CTA + static stress-CTP in detecting functionally significant CAD using invasive FFR as the reference standard. The study enrolled 147 consecutive symptomatic patients, and found that complementary functional information obtained by either CT-FFR or CTP significantly improves diagnostic performance, but no significant difference was detected between CTA + CT-FFR versus CTA + CTP [46]. Even though both CT-FFR and CT + CTP yielded statistically similar diagnostic performance, combined CT and static or dynamic CTP might provide additive diagnostic value in patients with inconclusive CT-FFR due to the higher specificity and positive predictive value [47]. CT-FFR provides information on the lesion-specific ischemia and is derived from rest CTA images therefore highly dependent on image quality. Dynamic CTP on the other hand could quantify total myocardial burden and thus might be useful in patients with extensive CAD or microvascular disease.

Growing body of evidence suggests that using CT for the detection of myocardial perfusion defects is feasible and reliable compared to other non-invasive and invasive methods. However, the lack of standardized methods regarding image acquisition, stress protocols and particularly image analysis limits its widespread use. Moreover, there is no unified MBF cutoff value for the detection of ischemic myocardium. Although the number of CTP tests is increasing as part of routine clinical assessment of intermediate lesions, there are limited data on the clinical value of CTP in specific patient populations prone to have ischemic heart disease.

\section{Potential role of myocardial perfusion imaging by $\mathrm{CT}$ in clinical practice}

Studies examining the potential role of CTP in clinical practice are scarce. In the evaluation of ischemic heart disease using additional stress CTP has a higher sensitivity compared to anatomical assessment alone mainly due to the reduction of false positive findings. Therefore, CTA could become a more robust gatekeeper by reducing unnecessary invasive procedures. Table 2 summarizes the potential role of CTP for a wide spectrum of ischemic heart disease patients.

\section{Stable angina}

Myocardial perfusion imaging by CT can improve patient management in stable angina. In the multicenter prospective CRESCENT-II (Comprehensive Cardiac CT Versus Exercise Testing in Suspected Coronary Artery Disease 2, NCT02291484) trial a diagnostic pathway including CTP was compared with traditional functional testing in patients with stable chest pain and found to be an effective and safe alternative for the diagnosis of CAD by lowering rates of ICA without coronary revascularization [48]. Similarly, in another study including 240 consecutive patients, adding dynamic CTP to CTA significantly reduced the number of unnecessary invasive testing in patients with intermediate risk for CAD (CTA only: 50.0\% [29/58] versus CTA + CTP: $10.8 \%$ [4/37], $P<0.0001)$ [49]. Myocardial ischemia was determined as $\mathrm{MBF} \leq 100 \mathrm{~mL} / 100 \mathrm{~mL} / \mathrm{min}$. In addition, van Rosendael et al. found a five-fold reduction of performing ICA in patients with obstructive CAD in case of normal myocardial perfusion on static stress CTP images [50].

Patients with extensive coronary calcification detected on native CT scans could be an optimal target for stress CTP. In challenging cases such as severe calcification (coronary calcium score $\geq 400$ ), combined CTA and CTP is superior to CTA alone by providing additional functional information 
Table 2. Potential clinical use of CTP in the spectrum of ischemic heart disease

Potential role of CTP

Stable angina

Acute chest pain

Post-myocardial infarction

Extensive non-obstructive CAD

Diabetes mellitus and arterial hypertension
Improve patient management in stable angina by detecting ischemic segments. Better gatekeeper function of FFR by reducing unnecessary invasive procedures.

Superior spatial resolution as compared with SPECT.

Useful in patients with extensive calcification (blooming artifacts).

Multivessel CAD evaluation to select vessel(s) for revascularization (dynamic CTP).

Prognostic information provided by combining plaque burden and ischemic load.

Ischemia detection in the presence of previously implanted stents. Evaluation of microvascular dysfunction using parameters of myocardial tissue hemodynamics. Exclude obstructive CAD in patients with low risk for ACS.

Improve risk prediction in patients after MI by evaluating infarcted territories. Global ischemia detection by quantitative CTP parameters. Early identification of epicardial coronary atherosclerosis, microvascular dysfunction and impaired myocardial perfusion in patients at higher risk.

ACS: acute coronary syndrome; CAD: coronary artery disease; CCTA: coronary CT angiography; CTP: CT perfusion; FFR: fractional flow reserve; MI: myocardial infarction; SPECT: single-photon emission computed tomography.

of coronary lesions $(\mathrm{AUC}=0.97$ versus $\mathrm{AUC}=0.088, P=$ $0.030)$. However, in patients with high pretest probability or known CAD combining CTA and CTP did not improve diagnostic performance in case of low Agatston calcium score (coronary calcium score < 400) [51].

More recent studies also examined the impact of MBF as assessed by dynamic CTP on clinical outcomes and found that it has an additive prognostic value over coronary CTA and is an independent predictor of major adverse cardiovascular events (MACEs). Using dynamic CTP, a summed stress score based on MBF values proved to be prognostically relevant after adjusted for obstructive CAD on CTA with hazard ratio (HR) of 5.7 (CI: 1.9-16.9, $P=0.002$ ) [52]. Also, myocardial perfusion defect defined by low index-MBF $(<0.88)$ calculated as a ratio between each segment and global MBF values was associated with MACE after adjusting for coronary CTA, CT-FFR findings, age, sex, and risk factors (HR: 10.1, CI: 2.1-48.8, $P=0.004$ ) [53]. Notably, dynamic CTP derived perfusion parameters had additive prognostic value beyond clinical risk factors and stenosis severity assessed by coronary CTA [54]. Moreover, the extent of myocardial perfusion defect was also a prognosticator of MACE.
Currently, two large, randomized trials aimed to evaluate the clinical utility and prognostic value of CTP. The CTPPRO (Impact of Stress Cardiac Computed Tomography Myocardial Perfusion on Downstream Resources and Prognosis in Patients with Suspected or Known Coronary Artery Disease, NCT03976921) study is an international, multicenter, prospective, open-label, randomized trial focusing on the cost-effectiveness of combined CTA + CTP strategy versus usual care in 2,000 intermediate-high risk patients with suspected or known CAD [55]. The PERFUSE RCT (Prospective Evaluation of Myocardial Perfusion Computed Tomography Trial, NCT02208388) sought to investigate the safety and effectiveness of CTP guided revascularization versus FFR guided revascularization in 1,000 patients with suspected stable CAD.

Besides the assessment of epicardial coronary arteries, CTP allows for the detection of microvascular dysfunction, although regarding data are scarce. Coronary flow reserve can also be accurately evaluated using specific dynamic CTP protocols in rest and stress phase [56]. In a recent study, quantified MBF using dynamic CTP also appeared to be significantly lower in myocardial segments affected by microvascular obstruction and had excellent diagnostic 
accuracy compared to the reference CMR data (AUC = 0.996, $P<0.001$ ) [57].

A sub-study of the CORE320 prospective trial divided patients into 3 groups based on the presence of obstructive $\mathrm{CAD}$ or perfusion defect defined by either coronary CTA and CTP or ICA and SPECT. Patients with ischemia but no obstructive stenosis (INOCA) were identified using CTA and static CTP. Interestingly, higher prevalence of positive remodeling, greater total and low attenuation atheroma volume was present in the INOCA group as compared to patients without either obstructive CAD or ischemia [58].

\section{CTP after coronary intervention}

Although only few studies are available, the use of CTP could also be beneficial in patients with prior coronary intervention for detecting residual ischemia. Due to the metallic artifacts the evaluation of coronary stents by CTA is limited. Adding stress CTP to rest CTA aids the diagnosis of in-stent-restenosis and helps the identification of patients who need further intervention (AUC $=0.82$ for CTP and CTA versus $\mathrm{AUC}=0.69$ for CTA alone, $P<0.001$ ) [59].

\section{Acute chest pain}

For patients presenting with acute on-set chest pain with low risk for acute coronary syndrome (ACS) coronary CTA is a reliable tool to exclude obstructive CAD [60]. The use of CTA can result in decreased time to diagnosis and to discharge, however it could also drive revascularization rates higher. In the CATCH-2 (Cardiac CT in the Treatment of Acute Chest Pain 2, NCT02014311) randomized controlled trial combined CTA + CTP was compared to CTA alone for the diagnosis and treatment of ischemic heart disease. The trial included patients with acute chest pain after ACS was ruled out by standard care. The combined protocol decreased the number of further invasive examinations and helped in guiding patient management [61]. In addition, in a similar patient population visual and semi-quantitative perfusion defect assessed by static CTP was associated with MACE with a median follow-up of 19 months independently of the patient's pretest probability. For visual perfusion defect the adjusted hazard ratio was 39 (CI: 11-134, $P<$ 0.0001 ), while for impaired perfusion based on TPR (cut-off 0.89 ) hazard ratio was 0.99 (CI: 0.98-0.99, $P<0.0001$ ). Moreover, poor prognosis was related to cases where $>10 \%$ of left ventricular myocardium was affected [62].

\section{Post-myocardial infarction patients}

It has been suggested that the evaluation of infarcted area after acute myocardial infarction (AMI) could improve risk prediction, as irreversible myocardial damage is a strong long-term prognosticator. Analyzing irreversibly damaged myocardial areas is feasible using CTP parameters [63]. Nakauchi et al. quantified MBF parameters using dynamic CTP with deconvolution analysis in patients after AMI and measured significant differences in tissue blood flow and blood volume between infarcted and non-infarcted myocardial territories (mean MBF: 51.96 versus $108.84 \mathrm{~mL} /$ $100 \mathrm{~g} / \mathrm{min}, P<0.01$ ) [64]. In patients with ST-segment elevation myocardial infarction and successful revascularization Pan et al. found that MBF derived from dynamic CTP significantly and inversely correlated with peak Troponin $T$ levels $(r=-0.682, P<0.001)$ and left ventricular function at 6 months $(r=0.585, P=0.001)$ [65].

\section{Extensive non-obstructive CAD}

In cases of large atherosclerotic plaque burden without obstructive stenosis the assessment of ischemia by CT could help in identifying patients at highest cardiovascular risk. Meinel et al. demonstrated that dynamic CTP is suitable for the evaluation of global myocardial perfusion parameters [66]. Global MBF, MBV, and volume transfer constant $\left(K_{\text {trans }}\right)$ were analyzed and correlated well with the severity and extent of CAD on coronary CTA and visual perfusion defects. In addition, after a follow up of 6,12 , and 18 months MBF predicted cardiovascular outcomes with a two-fold increased risk for major events in case of global $\mathrm{MBF}<121$ $\mathrm{mL} / 100 \mathrm{~mL} / \mathrm{min}$ [67]. Global MBF also showed incremental prognostic value over age, gender, clinical risk factors and stenosis severity on coronary CTA.

\section{Diabetes mellitus and arterial hypertension}

Dynamic CTP provides global quantification of LV myocardium that could improve early recognition of developing ischemic heart disease in different conditions such as diabetes mellitus or hypertension. Using dynamic CTP lower global perfusion values were found in patients with hypertension and diabetes mellitus with suspected CAD (hypertension versus normotension: MBV $18.5 \pm 3.0$ versus $19.7 \pm 2.3 \mathrm{~mL} / 100 \mathrm{~mL}, P<0.05$; diabetes versus no diabetes: MBV $17.9 \pm 2.4$ versus $19.4 \pm 2.8 \mathrm{~mL} / 100 \mathrm{~mL}, P<$ 0.05) [68]. Furthermore, longer duration of diabetes was associated with lower MBF assessed by dynamic CTP, yearly $6 \%$ additional risk was detected for decreased MBF [69]. Further larger studies are needed to establish the role of CTP in the diagnosis of common comorbidities such as diabetes and hypertension.

\section{Conclusion and future perspectives}

CTP is a promising tool for the identification of the presence and severity of perfusion abnormalities using either qualitative or quantitative analysis in patients with stable or even acute chest pain. Patients with extensive CAD and diffuse calcifications are optimal candidates to further improve the gatekeeper functionality of CTA. CTP can substantially increase the diagnostic accuracy of coronary CTA to identify possible candidates for revascularization and aggressive secondary prevention. However, differences in image acquisition protocols, image analysis, and artifacts limit the widespread clinical use of CTP. Advancements in scanner technology, post-processing software, and image reconstruction may help 
overcome the limitations of CTP. Also, further studies are warranted to establish proper indications for patients, who could benefit the most from CTP imaging (i.e., patients with diabetes, post-PCI, multivessel-disease, microvascular dysfunction). Furthermore, spectral CT imaging might help detect true perfusion defects using iodine density reconstructions in the future. Also, radiomic and machine learning analysis of the left ventricle may help overcome the limitations of CTP interpretation, especially the subjectivity of visual assessment. Furthermore, radiomics and machine learning may help in identifying new imaging biomarkers of the myocardium from CTP scans which may better identify myocardial injury and assist clinical decision making.

Funding sources: The project was supported by the KH-17 Programme of the National Research, Development and Innovation Office of the Ministry of Innovation and Technology in Hungary (NKFIH).

This study was supported by the National Research, Development and Innovation Office of Hungary (NKFIA; NVKP_16-1-2016-0017 National Heart Program). The research was financed by the Thematic Excellence Programme (Tématerületi Kiválósági Program, 2020-4.1.1.-TKP2020) of the Ministry for Innovation and Technology in Hungary, within the framework of the Therapeutic Development and Bioimaging programmes of the Semmelweis University.

The project was supported by the "NTP-NFTÖ" (Nemzeti Tehetség Program, Nemzet Fiatal Tehetségeiért Ösztöndíj) program of the Ministry of Human Capacities in Hungary (EMMI).

Bálint Szilveszter was supported by the ÚNKP-2020/21-4 Grant.

Authors' contribution: All authors reviewed the final version of the manuscript and agreed to submit it to IMAGING for publication.

Conflict of interest: The authors have no conflict of interest to disclose.

\section{REFERENCES}

[1] Patel MR, Peterson ED, Dai D, Brennan JM, Redberg RF, Anderson HV, et al.: Low diagnostic yield of elective coronary angiography. N Engl J Med 2010; 362(10): 886.

[2] Maurovich-Horvat P, Ferencik M, Voros S, Merkely B, Hoffmann $\mathrm{U}$ : Comprehensive plaque assessment by coronary CT angiography. Nat Rev Cardiol 2014; 11(7): 390.

[3] Yang L, Zhou T, Zhang R, Xu L, Peng Z, Ding J, et al.: Metaanalysis: diagnostic accuracy of coronary $\mathrm{CT}$ angiography with prospective ECG gating based on step-and-shoot, Flash and volume modes for detection of coronary artery disease. Eur Radiol 2014; 24(10): 2345.

[4] Shaw LJ, Hausleiter J, Achenbach S, Al-Mallah M, Berman DS, Budoff MJ, et al.: Coronary computed tomographic angiography as a gatekeeper to invasive diagnostic and surgical procedures: results from the multicenter CONFIRM (Coronary CT Angiography Evaluation for Clinical Outcomes: an International Multicenter) registry. J Am College Cardiol 2012; 60(20): 2103.

[5] Moss AJ, Williams MC, Newby DE, Nicol ED: The updated NICE guidelines: cardiac $\mathrm{CT}$ as the first-line test for coronary artery disease. Curr Cardiovasc Imaging Rep 2017; 10(5): 15.

[6] Knuuti J, Wijns W, Saraste A, Capodanno D, Barbato E, FunckBrentano C, et al.: ESC guidelines for the diagnosis and management of chronic coronary syndromes: the task force for the diagnosis and management of chronic coronary syndromes of the European Society of Cardiology (ESC). Eur Heart J 2019; 41(3): 407.

[7] Investigators S-H, Newby DE, Adamson PD, Berry C, Boon NA, Dweck MR, et al.: Coronary CT Angiography and 5-Year Risk of Myocardial Infarction. N Engl J Med 2018; 379(10): 924.

[8] Douglas PS, Hoffmann U, Patel MR, Mark DB, Al-Khalidi HR, Cavanaugh B, et al.: Outcomes of anatomical versus functional testing for coronary artery disease. N Engl J Med 2015; 372(14): 1291.

[9] Hoffmann U, Ferencik M, Udelson JE, Picard MH, Truong QA, Patel MR, et al.: Prognostic value of noninvasive cardiovascular testing in patients with stable chest pain: insights from the PROMISE trial (prospective multicenter imaging study for evaluation of chest pain). Circulation 2017; 135(24): 2320.

[10] Tonino PA, De Bruyne B, Pijls NH, Siebert U, Ikeno F, van' t Veer $\mathrm{M}$, et al.: Fractional flow reserve versus angiography for guiding percutaneous coronary intervention. N Engl J Med 2009; 360(3): 213.

[11] Maron DJ, Hochman JS, Reynolds HR, Bangalore S, O’Brien SM, Boden WE, et al.: Initial invasive or conservative strategy for stable coronary disease. N Engl J Med 2020; 382(15): 1395.

[12] Iwasaki K: Myocardial ischemia is a key factor in the management of stable coronary artery disease. World J Cardiol 2014; 6(4): 130.

[13] Tonino PA, Fearon WF, De Bruyne B, Oldroyd KG, Leesar MA, Ver Lee PN, et al.: Angiographic versus functional severity of coronary artery stenoses in the FAME study fractional flow reserve versus angiography in multivessel evaluation. J Am College Cardiol 2010; 55(25): 2816.

[14] De Bruyne B, Pijls NH, Kalesan B, Barbato E, Tonino PA, Piroth $\mathrm{Z}$, et al.: Fractional flow reserve-guided PCI versus medical therapy in stable coronary disease. N Engl J Med 2012; 367(11): 991.

[15] Boden WE, O'Rourke RA, Teo KK, Hartigan PM, Maron DJ, Kostuk WJ, et al.: Optimal medical therapy with or without PCI for stable coronary disease. N Engl J Med 2007; 356(15): 1503.

[16] Curzen N, Rana O, Nicholas Z, Golledge P, Zaman A, Oldroyd K, et al.: Does routine pressure wire assessment influence management strategy at coronary angiography for diagnosis of chest pain?: the RIPCORD study. Circ Cardiovasc Interv 2014; 7(2): 248.

[17] Schuijf JD, Wijns W, Jukema JW, Atsma DE, de Roos A, Lamb HJ, et al.: Relationship between noninvasive coronary angiography with multi-slice computed tomography and myocardial perfusion imaging. J Am Coll Cardiol 2006; 48(12): 2508.

[18] Seitun S, De Lorenzi C, Cademartiri F, Buscaglia A, Travaglio N, Balbi M, et al.: CT myocardial perfusion imaging: a new frontier in cardiac imaging. Biomed Res Int 2018; 2018: 7295460.

[19] Hachamovitch R, Hayes SW, Friedman JD, Cohen I, Berman DS: Comparison of the short-term survival benefit associated with 
revascularization compared with medical therapy in patients with no prior coronary artery disease undergoing stress myocardial perfusion single photon emission computed tomography. Circulation 2003; 107(23): 2900.

[20] Seitun S, Castiglione Morelli M, Budaj I, Boccalini S, Galletto Pregliasco A, Valbusa A, et al.: Stress computed tomography myocardial perfusion imaging: a new topic in cardiology. Rev Esp Cardiol (Engl Ed) 2016; 69(2): 188.

[21] Techasith T, Cury RC: Stress myocardial CT perfusion: an update and future perspective. JACC Cardiovasc Imaging 2011; 4(8): 905.

[22] Baxa J, Hromadka M, Sedivy J, Stepankova L, Molacek J, Schmidt B, et al.: Regadenoson-stress dynamic myocardial perfusion improves diagnostic performance of CT angiography in assessment of intermediate coronary artery stenosis in asymptomatic patients. Biomed Res Int 2015; 2015: 105629.

[23] Bischoff B, Bamberg F, Marcus R, Schwarz F, Becker HC, Becker A, et al: Optimal timing for first-pass stress CT myocardial perfusion imaging. Int J Cardiovasc Imaging 2013; 29(2): 435.

[24] Cademartiri F, Seitun S, Clemente A, La Grutta L, Toia P, Runza G, et al.: Myocardial blood flow quantification for evaluation of coronary artery disease by computed tomography. Cardiovasc Diagn Ther 2017; 7(2): 129.

[25] Arnoldi E, Lee YS, Ruzsics B, Weininger M, Spears JR, Rowley CP, et al.: CT detection of myocardial blood volume deficits: dualenergy CT compared with single-energy CT spectra. J Cardiovasc Comput Tomogr 2011; 5(6): 421.

[26] George RT, Jerosch-Herold M, Silva C, Kitagawa K, Bluemke DA, Lima JA, et al.: Quantification of myocardial perfusion using dynamic 64-detector computed tomography. Invest Radiol 2007; 42(12): 815.

[27] Danad I, Szymonifka J, Schulman-Marcus J, Min JK: Static and dynamic assessment of myocardial perfusion by computed tomography. Eur Heart J Cardiovasc Imaging 2016; 17(8): 836.

[28] Pontone G, Baggiano A, Andreini D, Guaricci AI, Guglielmo M, Muscogiuri G, et al.: Dynamic stress computed tomography perfusion with a whole-heart coverage scanner in addition to coronary computed tomography angiography and fractional flow reserve computed tomography derived. JACC Cardiovasc Imaging 2019; 12(12): 2460.

[29] Rossi A, Dharampal A, Wragg A, Davies LC, van Geuns RJ, Anagnostopoulos C, et al.: Diagnostic performance of hyperaemic myocardial blood flow index obtained by dynamic computed tomography: does it predict functionally significant coronary lesions? Eur Heart J Cardiovasc Imaging 2014; 15(1): 85.

[30] Bamberg F, Marcus RP, Becker A, Hildebrandt K, Bauner K, Schwarz F, et al.: Dynamic myocardial CT perfusion imaging for evaluation of myocardial ischemia as determined by MR imaging. JACC Cardiovasc Imaging 2014; 7(3): 267.

[31] Bamberg F, Becker A, Schwarz F, Marcus RP, Greif M, von Ziegler F, et al.: Detection of hemodynamically significant coronary artery stenosis: incremental diagnostic value of dynamic CT-based myocardial perfusion imaging. Radiology 2011; 260(3): 689.

[32] Coenen A, Rossi A, Lubbers MM, Kurata A, Kono AK, Chelu RG, et al.: Integrating CT myocardial perfusion and CT-FFR in the work-up of coronary artery disease. JACC Cardiovasc Imaging 2017; 10(7): 760.

[33] Kono AK, Coenen A, Lubbers M, Kurata A, Rossi A, Dharampal A, et al.: Relative myocardial blood flow by dynamic computed tomographic perfusion imaging predicts hemodynamic significance of coronary stenosis better than absolute blood flow. Invest Radiol 2014; 49(12): 801.

[34] Rossi A, Wragg A, Klotz E, Pirro F, Moon JC, Nieman K, et al.: Dynamic computed tomography myocardial perfusion imaging: comparison of clinical analysis methods for the detection of vessel-specific ischemia. Circ Cardiovasc Imaging 2017; 10(4).

[35] Greif M, von Ziegler F, Bamberg F, Tittus J, Schwarz F, D’Anastasi $\mathrm{M}$, et al.: CT stress perfusion imaging for detection of haemodynamically relevant coronary stenosis as defined by FFR. Heart 2013; 99(14): 1004.

[36] Brusen R, Branch K: Myocardial CT perfusion: a review of current modalities, technology, and clinical performance. Current Cardiovasc Imaging Report 2017; 10(8): 25.

[37] Patel AR, Bamberg F, Branch K, Carrascosa P, Chen M, Cury RC, et al.: Society of cardiovascular computed tomography expert consensus document on myocardial computed tomography perfusion imaging. J Cardiovasc Comput Tomogr 2020; 14(1): 87.

[38] Kikuchi Y, Oyama-Manabe N, Naya M, Manabe O, Tomiyama Y, Sasaki T, et al.: Quantification of myocardial blood flow using dynamic 320-row multi-detector CT as compared with (1)(5)OH(2)O PET. Eur Radiol 2014; 24(7): 1547.

[39] Bettencourt N, Chiribiri A, Schuster A, Ferreira N, Sampaio F, Pires-Morais G, et al.: Direct comparison of cardiac magnetic resonance and multidetector computed tomography stress-rest perfusion imaging for detection of coronary artery disease. J Am College Cardiol 2013; 61(10): 1099.

[40] Ko BS, Cameron JD, Meredith IT, Leung M, Antonis PR, Nasis A, et al.: Computed tomography stress myocardial perfusion imaging in patients considered for revascularization: a comparison with fractional flow reserve. Eur Heart J 2012; 33(1): 67.

[41] Tamarappoo BK, Gutstein A, Cheng VY, Nakazato R, Gransar H, Dey D, et al.: Assessment of the relationship between stenosis severity and distribution of coronary artery stenoses on multislice computed tomographic angiography and myocardial ischemia detected by single photon emission computed tomography. J Nucl Cardiol 2010; 17(5): 791.

[42] George RT, Mehra VC, Chen MY, Kitagawa K, Arbab-Zadeh A, Miller JM, et al.: Myocardial CT perfusion imaging and SPECT for the diagnosis of coronary artery disease: a head-to-head comparison from the CORE320 multicenter diagnostic performance study. Radiology 2014; 272(2): 407.

[43] Cury RC, Kitt TM, Feaheny K, Blankstein R, Ghoshhajra BB, Budoff MJ, et al.: A randomized, multicenter, multivendor study of myocardial perfusion imaging with regadenoson CT perfusion vs single photon emission CT. J Cardiovasc Comput Tomogr 2015; 9(2): 103.

[44] Takx RA, Blomberg BA, El Aidi H, Habets J, de Jong PA, Nagel E, et al.: Diagnostic accuracy of stress myocardial perfusion imaging compared to invasive coronary angiography with fractional flow reserve meta-analysis. Circ Cardiovasc Imaging 2015; 8(1).

[45] Sorgaard MH, Kofoed KF, Linde JJ, George RT, Rochitte CE, Feuchtner G, et al.: Diagnostic accuracy of static CT perfusion for the detection of myocardial ischemia. A systematic review and meta-analysis. J Cardiovasc Comput Tomogr 2016; 10(6): 450.

[46] Pontone G, Baggiano A, Andreini D, Guaricci AI, Guglielmo M, Muscogiuri G, et al.: Stress computed tomography perfusion versus fractional flow reserve CT derived in suspected coronary 
artery disease: the perfection study. JACC Cardiovasc Imaging 2019; 12 (8 Pt 1): 1487.

[47] Baggiano A, Fusini L, Del Torto A, Vivona P, Guglielmo M, Muscogiuri G, et al.: Sequential strategy including FFRCT plus stress-CTP impacts on management of patients with stable chest pain: the stress-CTP RIPCORD study. J Clin Med 2020; 9(7).

[48] Lubbers M, Coenen A, Kofflard M, Bruning T, Kietselaer B, Galema $\mathrm{T}$, et al.: Comprehensive cardiac CT with myocardial perfusion imaging versus functional testing in suspected coronary artery disease: the multicenter, randomized CRESCENT-II trial. JACC Cardiovasc Imaging 2018; 11(11): 1625.

[49] Yu M, Shen C, Dai X, Lu Z, Wang Y, Lu B, et al.: Clinical outcomes of dynamic computed tomography myocardial perfusion imaging combined with coronary computed tomography angiography versus coronary computed tomography angiography-guided strategy. Circ Cardiovasc Imaging 2020; 13(1): e009775.

[50] van Rosendael AR, Dimitriu-Leen AC, de Graaf MA, van Zwet EW, Jukema JW, Bax JJ, et al.: Impact of computed tomography myocardial perfusion following computed tomography coronary angiography on downstream referral for invasive coronary angiography, revascularization and, outcome at 12 months. Eur Heart J Cardiovasc Imaging 2017; 18(9): 969.

[51] Sharma RK, Arbab-Zadeh A, Kishi S, Chen MY, Magalhaes TA, George RT, et al.: Incremental diagnostic accuracy of computed tomography myocardial perfusion imaging over coronary angiography stratified by pre-test probability of coronary artery disease and severity of coronary artery calcification: The CORE320 study. Int J Cardiol 2015; 201: 570.

[52] Nakamura S, Kitagawa K, Goto Y, Omori T, Kurita T, Yamada A, et al.: Incremental prognostic value of myocardial blood flow quantified with stress dynamic computed tomography perfusion imaging. JACC Cardiovasc Imaging 2019; 12 (7 Pt 2): 1379.

[53] van Assen M, De Cecco CN, Eid M, von Knebel Doeberitz P, Scarabello M, Lavra F, et al.: Prognostic value of CT myocardial perfusion imaging and CT-derived fractional flow reserve for major adverse cardiac events in patients with coronary artery disease. J Cardiovasc Comput Tomogr 2019; 13(3): 26.

[54] Meinel FG, Pugliese F, Schoepf UJ, Ebersberger U, Wichmann JL, Lo GG, et al.: Prognostic value of stress dynamic myocardial perfusion CT in a multicenter population with known or suspected coronary artery disease. AJR Am J Roentgenol 2017; 208(4): 761.

[55] Pontone G, De Cecco C, Baggiano A, Guaricci AI, Guglielmo M, Leiner $\mathrm{T}$, et al.: Design of CTP-PRO study (impact of stress Cardiac computed Tomography myocardial Perfusion on downstream resources and PROgnosis in patients with suspected or known coronary artery disease: a multicenter international study). Int J Cardiol 2019; 292: 253.

[56] Ho KT, Ong HY, Tan G, Yong QW: Dynamic CT myocardial perfusion measurements of resting and hyperaemic blood flow in low-risk subjects with 128-slice dual-source CT. Eur Heart J Cardiovasc Imaging 2015; 16(3): 300.

[57] Yu M, Chen X, Dai X, Pan J, Wang Y, Lu B, et al.: The value of low-dose dynamic myocardial perfusion CT for accurate evaluation of microvascular obstruction in patients with acute myocardial infarction. AJR Am Journal Roentgenol 2019; 213(4): 798.

[58] Schuijf JD, Matheson MB, Ostovaneh MR, Arbab-Zadeh A, Kofoed KF, Scholte A, et al.: Ischemia and no obstructive stenosis
(INOCA) at CT angiography, CT myocardial perfusion, invasive coronary angiography, and SPECT: the CORE320 study. Radiology 2020; 294(1): 61.

[59] Rief M, Zimmermann E, Stenzel F, Martus P, Stangl K, Greupner $\mathrm{J}$, et al.: Computed tomography angiography and myocardial computed tomography perfusion in patients with coronary stents: prospective intraindividual comparison with conventional coronary angiography. J Am College Cardiol 2013; 62(16): 1476.

[60] Linde JJ, Hove JD, Sorgaard M, Kelbaek H, Jensen GB, Kuhl JT, et al.: Long-term clinical impact of coronary CT angiography in patients with recent acute-onset chest pain: the randomized controlled CATCH trial. JACC Cardiovasc Imaging 2015; 8(12): 1404.

[61] Sorgaard MH, Linde JJ, Kuhl JT, Kelbaek H, Hove JD, Fornitz GG, et al.: Value of myocardial perfusion assessment with coronary computed tomography angiography in patients with recent acuteonset chest pain. JACC Cardiovasc Imaging 2018; 11(11): 1611.

[62] Linde JJ, Sorgaard M, Kuhl JT, Hove JD, Kelbaek H, Nielsen WB, et al.: Prediction of clinical outcome by myocardial CT perfusion in patients with low-risk unstable angina pectoris. Int J Cardiovasc Imaging 2017; 33(2): 261.

[63] Han R, Sun K, Lu B, Zhao R, Li K, Yang X: Diagnostic accuracy of coronary $\mathrm{CT}$ angiography combined with dual-energy myocardial perfusion imaging for detection of myocardial infarction. Exp Ther Med 2017; 14(1): 207.

[64] Nakauchi Y, Iwanaga Y, Ikuta S, Kudo M, Kobuke K, Murakami T, et al.: Quantitative myocardial perfusion analysis using multi-row detector CT in acute myocardial infarction. Heart 2012; 98(7): 566.

[65] Pan J, Yuan M, Yu M, Gao Y, Shen C, Wang Y, et al.: Myocardial blood flow quantified by low-dose dynamic CT myocardial perfusion imaging is associated with peak troponin level and impaired left ventricle function in patients with ST-elevated myocardial infarction. Korean J Radiol 2019; 20(5): 709.

[66] Meinel FG, Ebersberger U, Schoepf UJ, Lo GG, Choe YH, Wang Y, et al.: Global quantification of left ventricular myocardial perfusion at dynamic CT: feasibility in a multicenter patient population. AJR Am J Roentgenol 2014; 203(2): W174.

[67] Meinel FG, Wichmann JL, Schoepf UJ, Pugliese F, Ebersberger U, Lo GG, et al.: Global quantification of left ventricular myocardial perfusion at dynamic CT imaging: prognostic value. J Cardiovasc Comput Tomogr 2017; 11(1): 16.

[68] Vliegenthart R, De Cecco CN, Wichmann JL, Meinel FG, Pelgrim GJ, Tesche C, et al.: Dynamic CT myocardial perfusion imaging identifies early perfusion abnormalities in diabetes and hypertension: insights from a multicenter registry. J Cardiovasc Comput Tomogr 2016; 10(4): 301.

[69] Tomizawa N, Fujino Y, Kamitani M, Chou S, Yamamoto K, Inoh $\mathrm{S}$, et al.: Longer diabetes duration reduces myocardial blood flow in remote myocardium assessed by dynamic myocardial CT perfusion. J Diabetes Complication 2018; 32(6): 609.

[70] Bettencourt N, Ferreira ND, Leite D, Carvalho M, Ferreira WDS, Schuster A, et al.: CAD detection in patients with intermediatehigh pre-test probability: low-dose CT delayed enhancement detects ischemic myocardial scar with moderate accuracy but does not improve performance of a stress-rest CT perfusion protocol. JACC Cardiovasc Imaging 2013; 6(10): 1062.

[71] Rochitte CE, George RT, Chen MY, Arbab-Zadeh A, Dewey M, Miller JM, et al.: Computed tomography angiography and 
perfusion to assess coronary artery stenosis causing perfusion defects by single photon emission computed tomography: the CORE320 study. Eur Heart J 2014; 35(17): 1120.

[72] Andreini D, Mushtaq S, Pontone G, Conte E, Collet C, Sonck J, et al.: CT perfusion versus coronary $\mathrm{CT}$ angiography in patients with suspected in-stent restenosis or CAD progression. JACC Cardiovasc Imaging 2020; 13(3): 732.

[73] Delgado C, Vazquez M, Oca R, Vilar M, Trinidad C, Sanmartin M: Myocardial ischemia evaluation with dual-source computed tomography: comparison with magnetic resonance imaging. Rev Esp Cardiol (Engl Ed) 2013; 66(11): 864.

[74] Meinel FG, De Cecco CN, Schoepf UJ, Nance JW, , Jr., Silverman JR, Flowers BA, et al.: First-arterial-pass dual-energy CT for assessment of myocardial blood supply: do we need rest, stress, and delayed acquisition? Comparison with SPECT. Radiology 2014; 270(3): 708.

[75] Kim SM, Chang SA, Shin W, Choe YH: Dual-energy CT perfusion during pharmacologic stress for the assessment of myocardial perfusion defects using a second-generation dual-source CT: a comparison with cardiac magnetic resonance imaging. J Comput Assist Tomogr 2014; 38(1): 44.

[76] Ko SM, Park JH, Hwang HK, Song MG: Direct comparison of stress- and rest-dual-energy computed tomography for detection of myocardial perfusion defect. Int J Cardiovasc Imaging 2014; 30(Suppl 1): 41.

[77] Delgado Sanchez-Gracian C, Oca Pernas R, Trinidad Lopez C, Santos Armentia E, Vaamonde Liste A, Vazquez Caamano M, et al.: Quantitative myocardial perfusion with stress dual-energy CT: iodine concentration differences between normal and ischemic or necrotic myocardium. Initial experience. Eur Radiol 2016; 26(9): 3199.

[78] Nishiyama H, Tanabe Y, Kido T, Kurata A, Uetani T, Kido T, et al.: Incremental diagnostic value of whole-heart dynamic computed tomography perfusion imaging for detecting obstructive coronary artery disease. J Cardiol 2019; 73(5): 425.

[79] Yi Y, Xu C, Wu W, Wang Y, Li YM, Shen ZJ, et al.: Myocardial blood flow analysis of stress dynamic myocardial CT perfusion for hemodynamically significant coronary artery disease diagnosis: The clinical value of relative parameter optimization. J Cardiovasc Comput Tomogr 2020; 14(4): 314.

[80] Yang J, Dou G, He B, Jin Q, Chen Z, Jing J, et al.: Stress myocardial blood flow ratio by dynamic CT perfusion identifies hemodynamically significant CAD. JACC Cardiovasc Imaging 2020; 13(4): 966.

[81] Alessio AM, Bindschadler M, Busey JM, Shuman WP, Caldwell $\mathrm{JH}$, Branch KR: Accuracy of myocardial blood flow estimation from dynamic contrast-enhanced cardiac CT compared with PET. Circ Cardiovasc Imaging 2019; 12(6): e008323.

[82] Yi Y, Xu C, Wu W, Wang Y, Li YM, Ge YQ, et al.: Stress dynamic myocardial CT perfusion for symptomatic patients with intermediate- or high-risk of coronary artery disease: optimization and incremental improvement between the absolute and relative myocardial blood flow analysis. J Cardiovasc Comput Tomogr 2020; 14(5): 437.

[83] Li Y, Dai X, Lu Z, Shen C, Zhang J: Diagnostic performance of quantitative, semi-quantitative, and visual analysis of dynamic CT myocardial perfusion imaging: a validation study with invasive fractional flow reserve. Eur Radiol 2020; 31(1): 525-34. 